\title{
The genus Ochrotrichia Mosely (Trichoptera: Hydroptilidae) in Costa Rica, with the description of four new species
}

\author{
JOAQUIN BUENO-SORIA ${ }^{1} \&$ RALPH W. HOLZENTHAL ${ }^{2}$

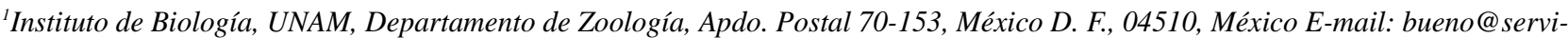 \\ dor.unam.mx \\ ${ }^{2}$ Department of Entomology, University of Minnesota, 219 Hodson Hall, 1980 Folwell Ave. St. Paul, MN 55108, U.S.A. E-mail: \\ holze001@umn.edu
}

\begin{abstract}
Four new species of the genus Ochrotrichia Mosely, are described from Costa Rica and the male genitalia figured (Ochrotrichia avicula new species, Ochrotrichia conformalis new species, Ochrotrichia jolandae new species, and Ochrotrichia quasi new species). Twenty-five species are recorded from Costa Rica, 8 for the first time [O. aldama (Mosely), O. arranca (Mosely), O. assita Bueno-Soria \& Holzenthal, O. caimita Flint, O. glabra Bueno-Soria \& Santiago-Fragoso, O. hondurenia Bueno-Soria \& Santiago-Fragoso, O. panamensis Flint, and O. tagala Flint] and new distributional records are listed. Ochrotrichia arranca (Mosely) is refigured and compared with $O$. conformalis, new species and $O$. quasi, new species.
\end{abstract}

Key words: Trichoptera, Hydroptilidae, Ochrotrichia, Costa Rica, new species, new records

\section{Introduction}

During the last 30 years, many new species of Ochrotrichia have been collected in Mexico, Central and South America, and the West Indies in a wide range of habitats, including tropical rain forest and seasonally dry habitats (Botosaneanu 1977, 1995, Bueno-Soria \& Santiago-Fragoso 1992, 1997, Bueno-Soria \& Holzenthal 1998, Denning \& Blickle 1972, Flint 1964, 1972, Flint \& Bueno-Soria 1999, Mosely1937). Flint et al. (1999) recorded 93 species in the genus for the Neotropics, including 8 species described by Wells \& Wichard (1989) in Dominican amber. Bueno-Soria \& Holzenthal (2004) recently added 21 additional new species for Mexico and Panama. It is not surprising that Costa Rica also harbors a rich Ochrotrichia fauna, composed now of 25 species (Table 1) (Bueno-Soria \& Santiago-Fragoso 1997, Bueno-Soria \& Holzenthal 1998, 2004, Flint 1972, Mosely 1937). Holzenthal and colleagues discovered this diversity largely in the late 1980s early 1990s during an intensive inventory of the Trichoptera of Costa Rica. In addition to the description of the 4 new species, we here provide new distributional records for the other 21 species from the country, including new country records. Also, the male genitalia of $O$. arranca (Mosely) are refigured and compared with $O$. conformalis, new species, and $O$. quasi, new species, because of the close similarity of these species.

All material will be deposited in the University of Minnesota Insect Collection, St. Paul, MN (UMSP), the National Museum of Natural History, Smithsonian Institution, Washington, D.C. (NMNH), the Instituto Nacional de Biodiversidad, Heredia, Costa Rica, (INBIO), the Colección Nacional de Insectos, Universidad Nacional Autónoma de México, Mexico City (CNIN), and the Carnegie Museum Collection, Pittsburgh, Pennsylvania (CMC), as indicated in the species descriptions and records. 
TABLE 1. Ochrotrichia of Costa Rica.

\begin{tabular}{|c|c|}
\hline Species & Note \\
\hline Ochrotrichia aldama (Mosely, 1937) & New country record \\
\hline Ochrotrichia arranca (Mosely, 1937) & New country record \\
\hline Ochrotrichia assita Bueno-Soria \& Holzenthal, 2004 & New country record \\
\hline Ochrotrichia avicula Bueno-Soria \& Holzenthal & New species \\
\hline \multicolumn{2}{|l|}{ Ochrotrichia avis Bueno-Soria \& Holzenthal, 1998} \\
\hline Ochrotrichia caimita Flint, 1972 & New country record \\
\hline Ochrotrichia conformalis Bueno-Soria \& Holzenthal & New species \\
\hline \multicolumn{2}{|l|}{ Ochrotrichia dulce Bueno-Soria \& Holzenthal, 1998} \\
\hline \multicolumn{2}{|l|}{ Ochrotrichia filiforma Flint, 1972} \\
\hline Ochrotrichia glabra Bueno-Soria \& Santiago-Fragoso, 1997 & New country record \\
\hline Ochrotrichia hondurenia Bueno-Soria \& Santiago-Fragoso, 1997 & New country record \\
\hline Ochrotrichia jolandae Bueno-Soria \& Holzenthal & New species \\
\hline \multicolumn{2}{|l|}{ Ochrotrichia longispina Bueno-Soria \& Holzenthal, 2004} \\
\hline \multicolumn{2}{|l|}{ Ochrotrichia membrana Bueno-Soria \& Holzenthal, 1998} \\
\hline \multicolumn{2}{|l|}{ Ochrotrichia moselyi Flint, 1972} \\
\hline \multicolumn{2}{|l|}{ Ochrotrichia pacifica Flint, 1972} \\
\hline Ochrotrichia panamensis Flint, 1972 & New country record \\
\hline Ochrotrichia quasi Bueno-Soria \& Holzenthal & New species \\
\hline \multicolumn{2}{|l|}{ Ochrotrichia quebrada Bueno-Soria \& Holzenthal, 1998} \\
\hline \multicolumn{2}{|l|}{ Ochrotrichia quinealensis Bueno-Soria \& Holzenthal, 1998} \\
\hline \multicolumn{2}{|l|}{ Ochrotrichia ramona Bueno-Soria \& Holzenthal, 1998} \\
\hline \multicolumn{2}{|l|}{ Ochrotrichia silva Bueno-Soria \& Holzenthal, 1998} \\
\hline Ochrotrichia tagala Flint, 1972 & New country record \\
\hline \multicolumn{2}{|l|}{ Ochrotrichia tenanga (Mosely, 1937) } \\
\hline Ochrotrichia vieja Bueno-Soria \& Holzenthal, 1998 & \\
\hline
\end{tabular}

\section{Species descriptions}

\section{Ochrotrichia avicula, new species}

Fig. 1a,b,c

This species is a member of the xena group of Flint (1972) as indicated by segment X occurring as a single broad, elongated plate. It is related to Ochrotrichia avis Bueno-Soria \& Holzenthal and O. quebrada BuenoSoria \& Holzenthal by the similarly elongated appearance of segment X in lateral view. From these species, O. avicula new species differs by the presence of a wide, straight, elongated spine on the left side of segment $\mathrm{X}$.

Male: Length of forewing $2.5 \mathrm{~mm}$. Color in alcohol dark brown. Segment IX depressed, slightly produced anteriad. Segment X in dorsal view hood-like, with wide, long, straight, spine-like process on left side; apex with short sclerotized hook-like on right side; in lateral view, hood-like with strong hooked apex curved ventrad. Inferior appendage in lateral view elongate, apex rounded; apical portion and midventral areas with group of black peg-like setae; in ventral view symmetrical, with group of large, black, peg-like setae apically and midventrally. Phallus long, tubular, with slightly wider basal section.

Female: Unknown

Type material: Holotype, male. COSTA RICA: Puntarenas: Río Jaba at rock quarry, $1.4 \mathrm{~km}$ (air) W Las Cruces, $8.79^{\circ} \mathrm{N}, 82.97^{\circ} \mathrm{W}$, el. 1150 m, 9.viii.1990, Holzenthal, Blahnik and Muñoz (UMSP). Paratypes: same data as the holotype 6 males (UMSP, NMNH, INBIO, CNIN).

Etymology: Avicula is the Latin feminine diminutive of bird, referring to the beak-like apex of segment $\mathrm{X}$ in lateral view. 

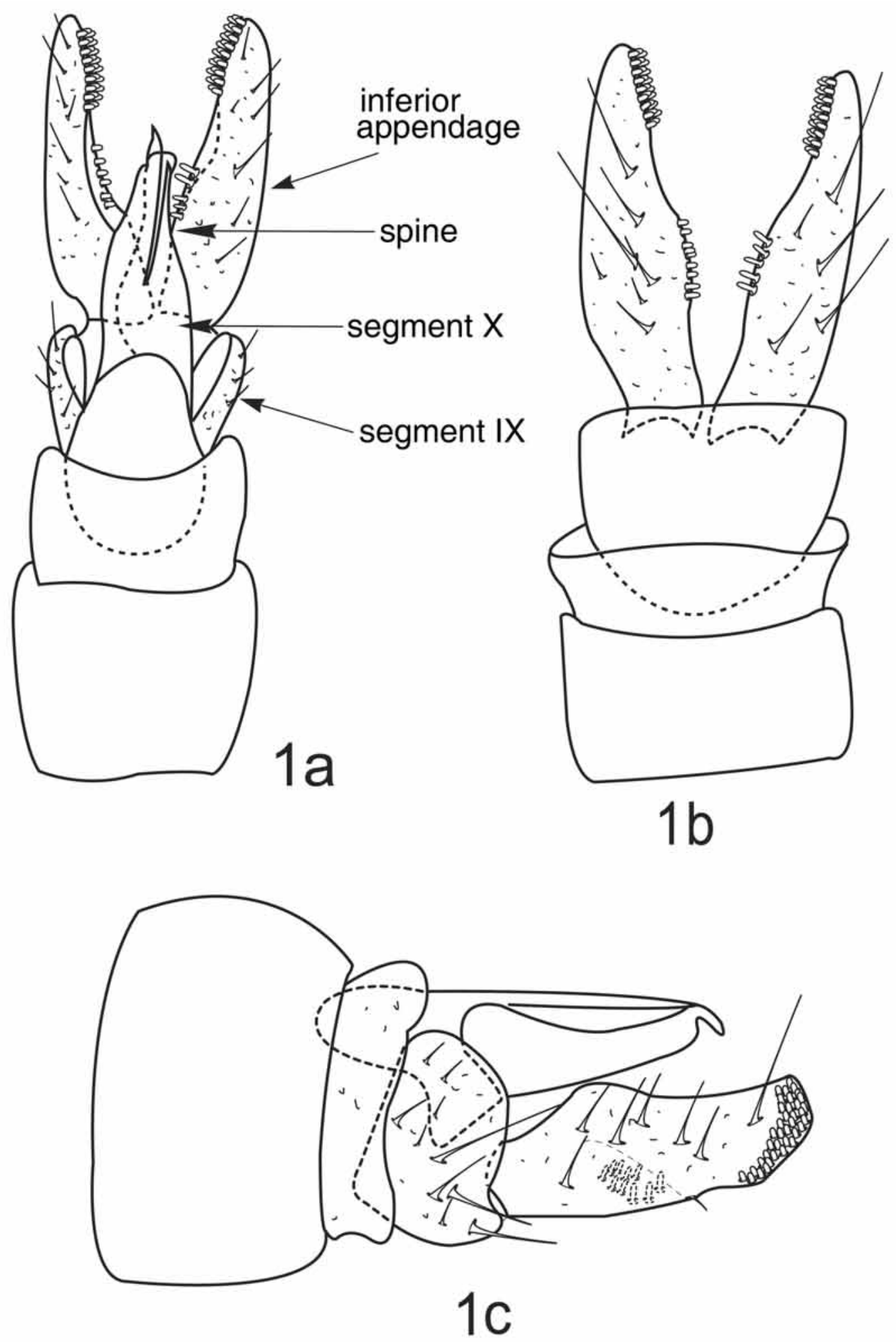

FIGURE 1. Ochrotrichia avicula new species. Male genitalia. 1a, dorsal view. 1b, ventral view. 1c, left lateral view.

\section{Ochrotrichia jolandae, new species}

Figs. 2a, b, c, d

This species appears to be another member of the xena group, perhaps related to Ochrotrichia concha BuenoSoria \& Santiago-Fragoso by the similar trumpet shape of the inferior appendages. However, $O$. jolandae new 
species is characterized by the stout, basal, spine on segment $X$ and the long spine-like process on the left side surrounded preapically by a long, hook-like, ventrally directed process.

Male: Length of forewing $2.5 \mathrm{~mm}$. Color in alcohol dark stramineous. Segment IX with posterodorsal margin slightly produced. Segment $X$ in dorsal view a long, narrow, plate with stout, basal spine-like on right side and long, straight spine-like process with apex curved to right, surrounded preapically by long, hook-like process; apical region narrow, slightly curved to right; in left lateral view appears with short, stout basal spinelike and long, straight, spine-like process, surrounded by stout, strongly curved ventrad hook-like process at midlength. Inferior appendages symmetrical, in lateral view trumpet shaped with apex and midventral margin rounded, with band of black peg-like setae, with stout, short, spine-like corner on midventral margin. Phallus arising from conical expanded base, apical region slender with sagittate process.

Female: Unknown

Type material: Holotype, male. COSTA RICA: Alajuela: Reserva Forestal San Ramón Río San Lorencito and tribs., $10.216^{\circ} \mathrm{N}, 84.607^{\circ} \mathrm{W}$, el. $980 \mathrm{~m}, 6-10.1 i i .1991$, Holzenthal, Muñoz, Huisman (UMSP). Paratype: Same data as holotype, 1 male (UMSP).

Etymology. We are pleased to dedicate this species to our colleague Jolanda Huisman.

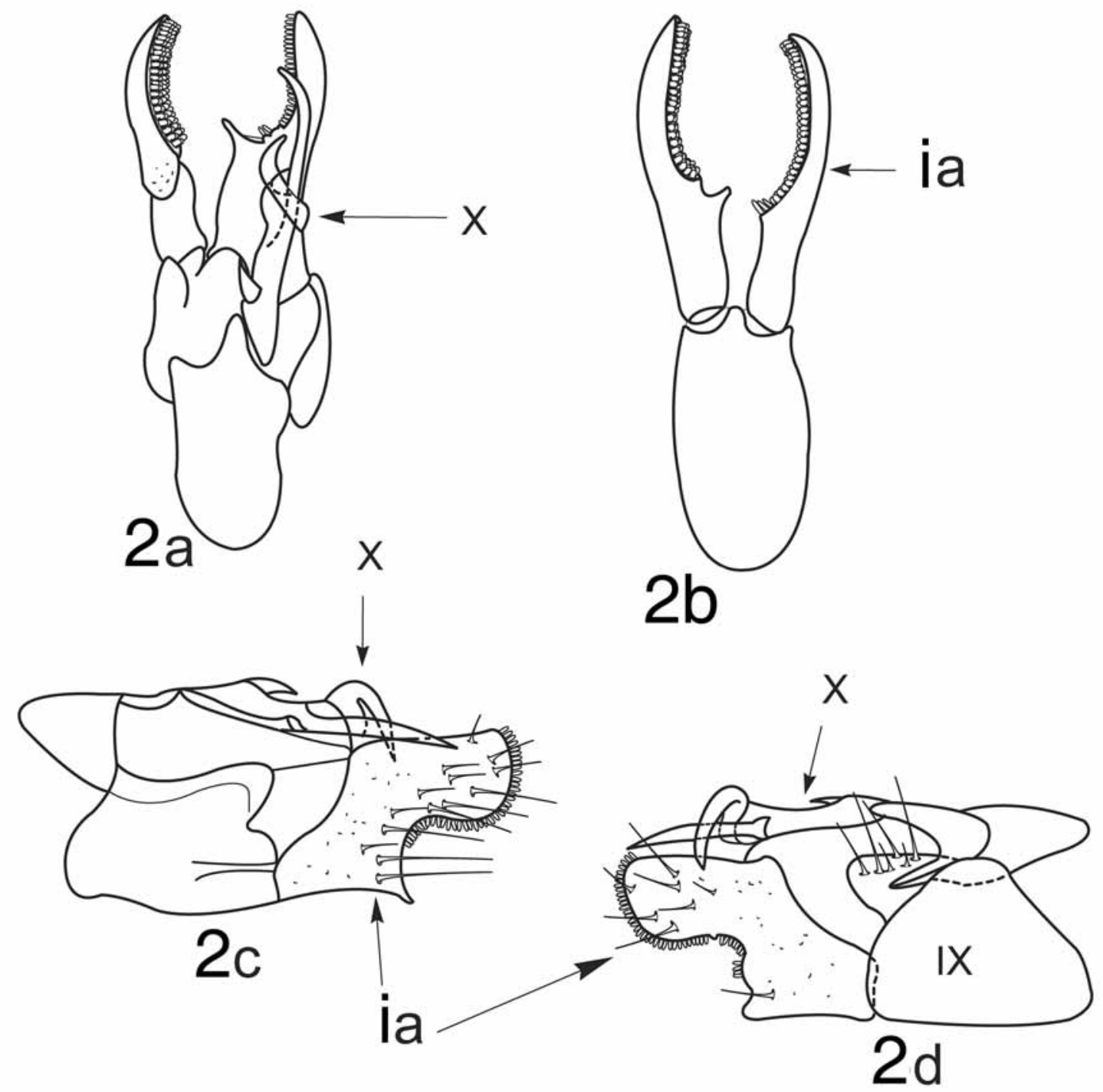

FIGURE 2. Ochrotrichia jolandae new species. Male genitalia. $2 \mathrm{a}$, dorsal view. $2 \mathrm{~b}$, ventral view. $2 \mathrm{c}$, left lateral view. 2d, right lateral view. Abbreviations: Inferior appendages (ia). 


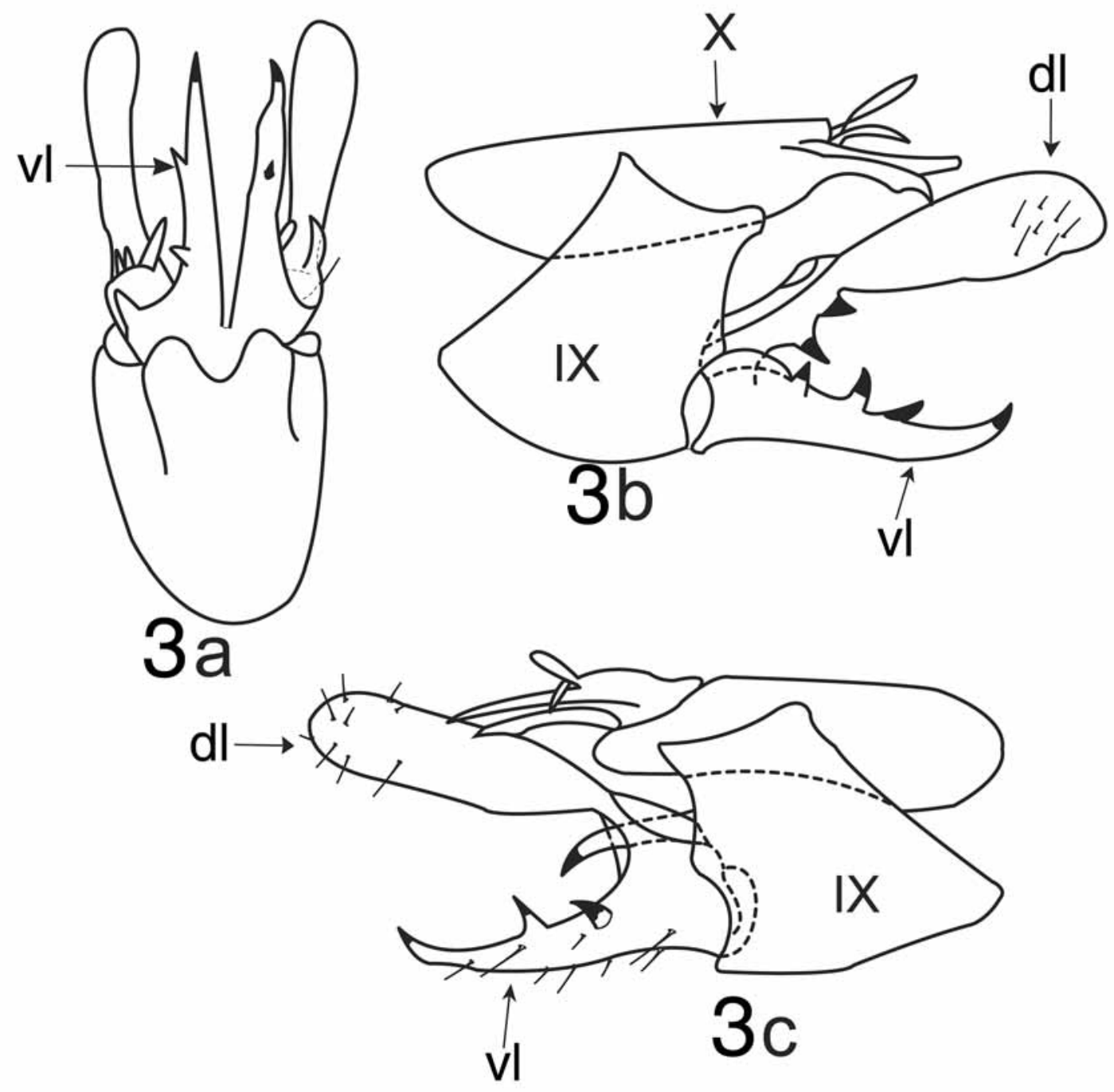

FIGURE 3. Ochrotrichia arranca (Mosely). Male genitalia. 3a, ventral view. 3b, left lateral view. 3c, right lateral view. Abbreviations: segment IX (IX), segment X (X), Dorsal lobe (dl), Ventral lobe (vl).

\section{Ochrotrichia conformalis, new species}

Figs. 4a, b, c

The presence of 2 dark spine-like processes on segment $X$ and similarly shaped inferior appendages in lateral view, make to Ochrotrichia conformalis new species a member of the arranca group (Flint 1972) closely related to O. arranca (Mosely) (Fig. 3), and O. quasi, new species. However, the wide, stout, basal lobe of the inferior appendages in ventral view clearly separates $O$. conformalis new species from $O$. arranca (Mosely) and $O$. quasi, new species.

Male: Length of forewing $2.0 \mathrm{~mm}$. Color in alcohol stramineous. Segment IX depressed, produced anteriad. Segment X in dorsal view with long, slightly curved, dorsal process bearing 2 large black setae; left side with apical hook-like process; in lateral view, ventrally with 2 curved processes, 1 long with acute, ventrally directed apex, the other shorter, wider basally. Inferior appendage in lateral view, with elongate, finger-like dorsal lobe; left inferior appendage with elongate, bifurcate ventral lobe, between lobes 2 short black spineslike; right inferior appendage with large, apically darkened hook-like process, ventral lobe bearing 2 preapical, black spines-like; in ventral view, left and right ventral lobes of inferior appendages appearing short, stout, wide basally, with 2 dark, preapical spines-like; apex acute, ending in long dark spine-like. Phallus long, thread-like. 
Female: Unknown

Type material: Holotype, male. COSTA RICA: Alajuela: Reserva Forestal San Ramón, Río San Lorencito and tribs., $10.216^{\circ} \mathrm{N}, 84.607^{\circ} \mathrm{W}$, el. $980 \mathrm{~m}, 1-4 . v .1990$, Holzenthal \& Blahnik (UMSP). Paratypes: same data as holotype, 2 males (UMSP, NMNH).

Etymology: From the Latin word conformalis; in reference to the similarity of this species with $O$. arranca (Mosely).
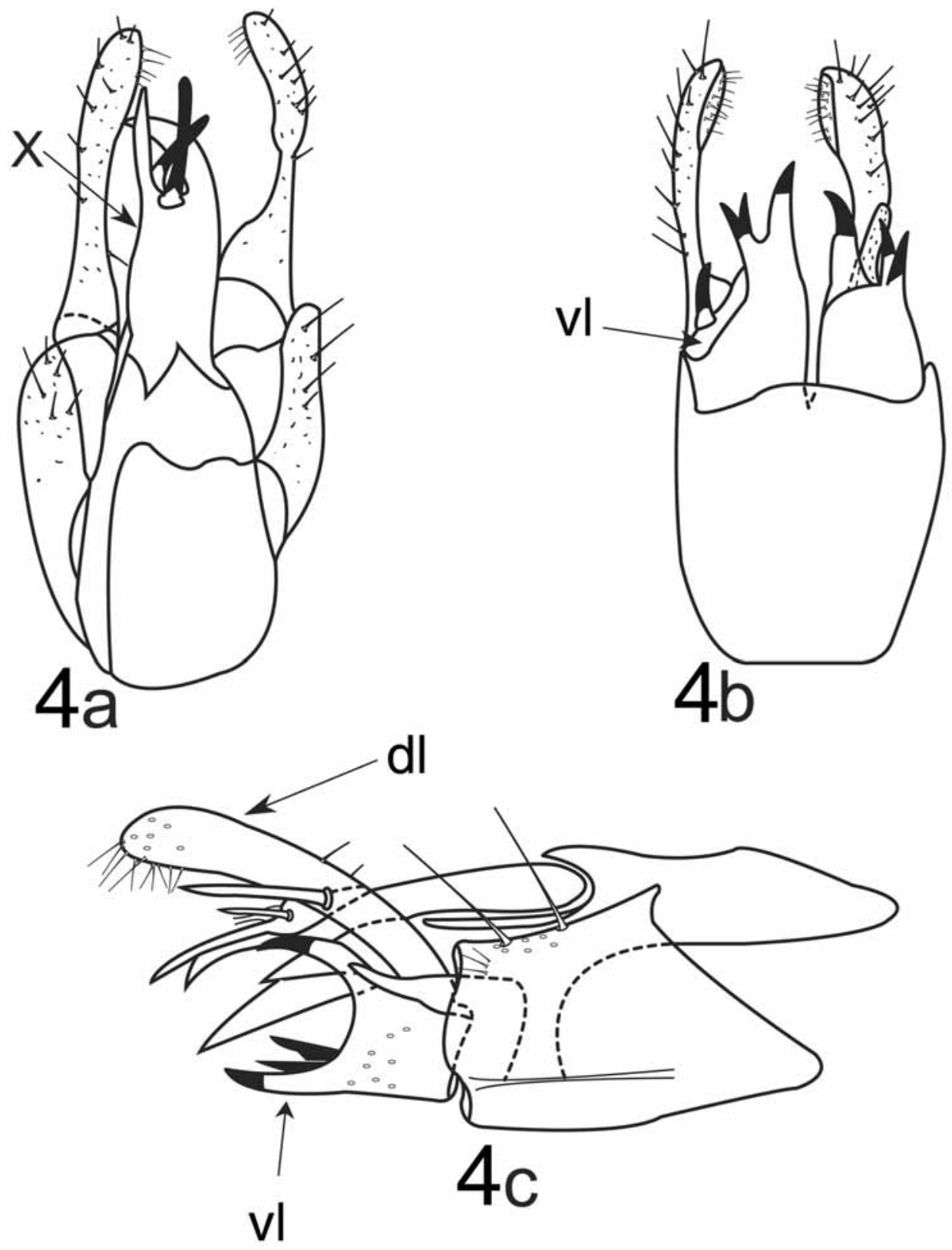

FIGURE 4. Ochrotrichia conformalis new species. Male genitalia. 4a, dorsal view. 4b, ventral view. 4c, right lateral view.

\section{Ochrotrichia quasi, new species}

Figs. 5a, b, c

Based on the finger-like dorsal lobe of the inferior appendages, this is another species of the arranca group closely related to Ochrotrichia arranca (Mosely) and O. conformalis new species. However, the distribution 
of spines on the inferior appendage and small size of its ventral lobe, clearly separate $O$. quasi new species from $O$. arranca (Mosely) and $O$. conformalis new species.

Male: Length of forewing $2.0 \mathrm{~mm}$. Color in alcohol stramineous. Segment IX depressed, slightly produced anteriad, with posterodorsal margin slightly produced. Segment X in dorsal view, with long, slightly curved, dorsal process, bearing 2 large black setae; in lateral view, left side with apically bifurcated process with acute apex. Inferior appendage with elongate, finger-like, dorsal lobe; on right side with strong spine-like process on base of dorsal lobe; left inferior appendage with elongate ventral lobe; between lobes dark apical spines-like of 2 different sizes; in ventral view, ventral lobe with pair of short, stout, preapical spine-like processes. Phallus long, thread-like.

Female: Unknown

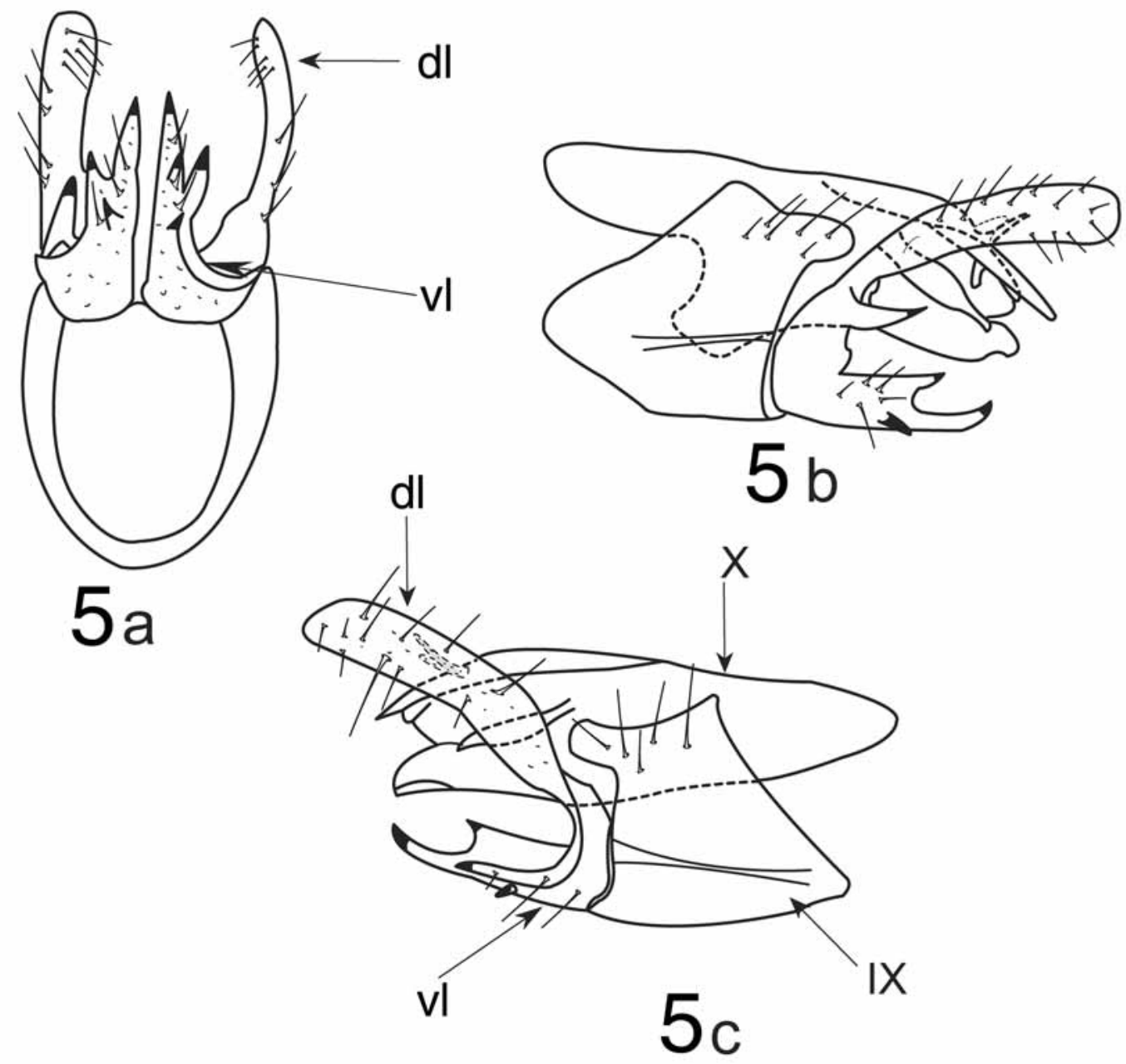

FIGURE 5. Ochrotrichia quasi new species. Male genitalia. $5 \mathrm{a}$, ventral view. $5 \mathrm{~b}$, left lateral view. $5 \mathrm{c}$, right lateral view.

Type material: Holotype, male. COSTA RICA: San José: Río Savegra, San Gerardo de Dota $9.33^{\circ}$ N, 83.48 W, el. 2200 m, 9.viii.1995, C. Young (CMC). Paratypes: Same data as the holotype but 9.viii.1995, 1 male (NMNH), 4.iii.1995, 1male (UMSP).

Etymology: Quasi is the Latin word meaning "appearing as if;" referring to the similarity of this species with $O$. conformalis new species and $O$. arranca (Mosely). 


\section{New Records of Costa Rican Ochrotrichia}

\section{Ochrotrichia aldama (Mosely). NEW COUNTRY RECORD}

Polytrichia aldama Mosely 1937:185

The type species was described from Chiapas, Mexico, and it has been reported from Dominican amber by Wells and Wichard (1989).

COSTA RICA: Alajuela: Río Pizote, ca $5 \mathrm{~km}$ (air) S Brasilia, $10.972^{\circ} \mathrm{N}, 85.345^{\circ} \mathrm{W}$, el. $390 \mathrm{~m}$, 12.iii.1986, Holzenthal \& Fasth, 3 males (INBIO); Río Pizote, ca 5 km N Dos Ríos, $10.948^{\circ} \mathrm{N}, 85.291^{\circ} \mathrm{W}$, el. 470 m, 9.iii.1986, Holzenthal \& Fasth, 2 males (NMNH); Reserva Forestal San Ramón, Río San Lorencito and tribs., $10.216^{\circ} \mathrm{N}, 84.607^{\circ} \mathrm{W}$, el. $980 \mathrm{~m}, 1-4 . v .1990$, Holzenthal \& Blahnik, 1 male (UMSP); Quebrada Honda 5.4 km, (road) S Crucera, $10.311^{\circ} \mathrm{N}, 84.245^{\circ} \mathrm{W}$, el. $650 \mathrm{~m}$, 12.ii.1992, Holzenthal Muñoz \& Kjer, 1 male (UMSP); Guanacaste: Quebrada García 10.6 km ENE Quebrada Grande, $10.862^{\circ} \mathrm{N}, 85.428^{\circ} \mathrm{W}$, el. 470 m, 8.iii.1986, Holzenthal \& Fasth, 1 male (UMSP); Río Tizate, $7.2 \mathrm{~km}$ NE Cañas Dulces, $10.773^{\circ} \mathrm{N}, 85.449^{\circ}$ W, el. 275 m, 28.vi.1986, Holzenthal, Heyn \& Armitage, 2 males (UMSP); Limón: Río Telire and small trib.,

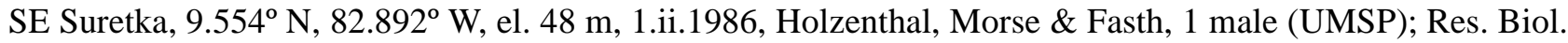
Barbilla, Río Dantas, 15 km S Pacuarito, 9.994 N, 83.443 W, el. 300 m, 27-30.i.1992, Holzenthal, Muñoz \& Kjer, 1 male (UMSP).

\section{Ochrotrichia arranca (Mosely). NEW COUNTRY RECORD}

(Figure 3)

Polytrichia arranca Mosely 1937:185

The type species was described from Chiapas, Mexico, and redescribed by Flint (1972).

COSTA RICA: Puntarenas: Zona Protectora Las Tablas, Río Cotón, Sitio Cotón, $8.941^{\circ} \mathrm{N}, 82.787^{\circ} \mathrm{W}$, el. 1460 m, 15.iv.1989, Holzenthal \& Blahnik, 1 male (UMSP); San José: Río Savegra @ San Gerardo de Dota, $9.33^{\circ} \mathrm{N}, 83.48^{\circ} \mathrm{W}$, el. $2200 \mathrm{~m}$, 9.viii.1995, C. Young, 1 male (CMC).

\section{Ochrotrichia assita Bueno-Soria \& Holzenthal. NEW COUNTRY RECORD}

Ochrotrichia assita Bueno-Soria \& Holzenthal 2004:251.

The type species was described from Chiriqui, Panama.

COSTA RICA: Heredia: Río Sarapiquí, $7 \mathrm{~km}$ W Puerto Viejo, $10.452^{\circ} \mathrm{N}, 84.067^{\circ} \mathrm{W}$, el. $50 \mathrm{~m}$, 11.ii.1986, Morse \& Fasth, 1 male (UMSP).

\section{Ochrotrichia avis Bueno-Soria \& Holzenthal}

Ochrotrichia avis Bueno-Soria \& Holzenthal 1998:606.

The type species was decribed from Alajuela, Costa Rica.

COSTA RICA: Alajuela: Reserva Forestal San Ramón, Río San Lorencito and tribs., $10.216^{\circ} \mathrm{N}, 84.607^{\circ} \mathrm{W}$, el. 980 m, 30.iii.-1.iv.1987, Holzenthal, Hamilton \& Heyn, 2 males (UMSP). 


\section{Ochrotrichia caimita Flint. NEW COUNTRY RECORD}

Ochrotrichia caimita Flint 1972:6.

The type species was described from Chiriqui, Panama.

COSTA RICA: Puntarenas: Río Singrí, ca 2 km (air) S Finca Helechales, $9.057^{\circ} \mathrm{N}, 83.082^{\circ} \mathrm{W}$, el. 720 m, 21.ii.1986, Holzenthal Morse \& Fasth, 3 males (UMSP), 2 males (INBIO); Río Guineal, ca. I km (air) E Finca Helechales, $9.076^{\circ} \mathrm{N}, 83.092^{\circ} \mathrm{W}$, el. 840 m, 22.ii.1986, Holzenthal Morse \& Fasth, 2 males (UMSP); Río Ceiba, route 2, ca. $6 \mathrm{~km} \mathrm{~W}$ road to Buenos Aires, $9.149^{\circ} \mathrm{N}, 83.377^{\circ} \mathrm{W}$, el. $250 \mathrm{~m}$, 20.ii.1986, Holzenthal, Morse \& Fasth, 1 male (NMNH).

\section{Ochrotrichia dulce Bueno-Soria \& Holzenthal}

Ochrotrichia dulce Bueno-Soria \& Holzenthal 1998:608.

The type species was described from Guanacaste, Costa Rica.

COSTA RICA: Guanacaste: Río Tizate, $7.2 \mathrm{~km}$ NE Cañas Dulces, $10.773^{\circ} \mathrm{N}, 85.449^{\circ} \mathrm{W}$, el. $275 \mathrm{~m}$, 28.vi.1986, Holzenthal, Heyn \& Armitage, 1 male (UMSP).

\section{Ochrotrichia filiforma Flint}

Ochrotrichia filiforma Flint 1972:9.

The type species was described from Cartago, Costa Rica.

COSTA RICA: Cartago: Chitaria, 19.vi.1967, Flint \& Ortiz, Type 72041 (NMNH); Río Platanillo 2.2 km E Tayutic, 9.82 ${ }^{\circ} \mathrm{N}, 83.55^{\circ} \mathrm{W}$, el. $730 \mathrm{~m}$, 30.i.1986, Holzenthal, Morse \& Fasth, 3 males (UMSP); Heredia: P. N. Braulio Carrillo, Est. El Ceibo, Río Peje, 10.327º N, 84.076º W, el. 480 m, 29-31.v.1990, Holzenthal, Blahnik \& Muñoz, 1 male (INBIO).

\section{Ochrotrichia glabra Bueno-Soria \& Santiago-Fragoso. NEW COUNTRY RECORD}

Ochrotrichia glabra Bueno-Soria \& Santiago-Fragoso 1997:364.

The type species was described from Chiriqui, Panama.

COSTA RICA: Alajuela: Reserva Forestal San Ramón, Río San Lorencito and tribs., $10.216^{\circ}$ N, $84.607^{\circ}$ W, el. 980 m, 1-4.v.1990, Holzenthal \& Blahnik, 1 male (UMSP).

\section{Ochrotrichia hondurenia Bueno-Soria \& Santiago-Fragoso. NEW COUNTRY RECORD}

Ochrotrichia hondurenia Bueno-Soria \& Santiago-Fragoso 1997:364.

The type species was described from Cayo District, Belize.

COSTA RICA: Guanacaste: Parque Nacional Guanacaste ca $7 \mathrm{~km}$ NE Maritza, $10.96^{\circ} \mathrm{N}, 85.50^{\circ} \mathrm{W}$, el. 550 m, 31.viii.1990, Huisman \& Quesada, 1 male (UMSP). 


\section{Ochrotrichia longispina Bueno-Soria \& Holzenthal}

Ochrotrichia longispina Bueno-Soria \& Holzenthal 2004:250.

The type species was described from Chiriqui, Panama.

COSTA RICA: Alajuela: Reserva Forestal San Ramón, Río San Lorencito and tribs., 10.216 ${ }^{\circ}$ N, $8^{4} .607^{\circ}$ W, el. 980 m, 30.iii.-1.iv.1987, Holzenthal, Hamilton \& Heyn, 1 male paratype (UMSP).

\section{Ochrotrichia membrana Bueno-Soria \& Holzenthal}

Ochrotrichia membrana Bueno-Soria \& Holzenthal 1998:604.

The type species was described from Alajuela, Costa Rica.

COSTA RICA: Alajuela: Reserva Forestal San Ramón, Río San Lorencito and tribs., 10.216 ${ }^{\circ}$ N, $8^{4} .607^{\circ}$ W, el. 980 m, 30.iii.-1.iv.1987, Holzenthal, Hamilton \& Heyn, 1 male (NMNH).

\section{Ochrotrichia moselyi Flint}

Ochrotrichia moselyi Flint 1972:7.

The type species was described from Veracruz, Mexico, and has been reported from Mexico,Guatemala and Costa Rica by Flint et al. (1999).

COSTA RICA: Alajuela: Río Pizote, ca $5 \mathrm{~km}$ (air) S Brasilia,10.972 ${ }^{\circ} \mathrm{N}, 85.345^{\circ} \mathrm{W}$, el. $390 \mathrm{~m}$, 12.iii.1986, Holzenthal \& Fasth, 1 male (UMSP); Río Pizote, ca $5 \mathrm{~km}$ N Dos Ríos, $10.948^{\circ} \mathrm{N}, 85.291^{\circ} \mathrm{W}$, el. 470 m, 9.iii.1986, Holzenthal \& Fasth, 1 male (UMSP); Guanacaste: Parque Nacional. Guanacaste, Estación Maritza, Río Tempisquito Sur, $10.95^{\circ} \mathrm{N}, 85.48^{\circ} \mathrm{W}$, el. 600 m, 30.viii.1990, Huismann \& Quesada, 1 male (UMSP).

\section{Ochrotrichia pacifica Flint}

Ochrotrichia pacifica Flint 1972:6.

The type species was described from Chiriqui, Panama.

COSTA RICA: Alajuela: Río Pizote, ca $5 \mathrm{~km}$ (air) S Brasilia, $10.972^{\circ} \mathrm{N}, 85.345^{\circ} \mathrm{W}$, el. $390 \mathrm{~m}$, 12.iii.1986, Holzenthal \& Fasth, 2 males (UMSP); Río Pizote, ca. 5 km N Dos Ríos, $10.948^{\circ} \mathrm{N}, 85.291^{\circ} \mathrm{W}$, el. 470 m, 9.iii.1986, Holzenthal \& Fasth, 3 males (UMSP); Guanacaste: Río Coribici, Las Cañas, 26.vii.1967, O. S. Flint Jr., 1 male (NMNH); Río Mena, $4.2 \mathrm{~km} \mathrm{~W} \mathrm{Santa} \mathrm{Cecilia,} 11.059^{\circ} \mathrm{N}, 85.448^{\circ} \mathrm{W}$, el. $260 \mathrm{~m}$, 11.iii.1986, Holzenthal \& Fasth, 6 males (INBIO); Heredia: Río Sarapiquí 7 km W Puerto Viejo, $10.452^{\circ} \mathrm{N}$, $84.067^{\circ} \mathrm{W}$, el. $50 \mathrm{~m}$, 11.ii.1986, Morse \& Fasth, 6 males (UMSP); Est. La Selva, Quebrada Sura, $10.437^{\circ} \mathrm{N}$, $84.010^{\circ} \mathrm{W}$, el. $50 \mathrm{~m}, 20-21 . v i .1986$, Holzenthal, Heyn \& Armitage, 5 males (UMSP); Est. Biol. La Selva, Río Puerto Viejo, $10.440^{\circ} \mathrm{N}, 84.012^{\circ} \mathrm{W}$, el. $30 \mathrm{~m}, 19$. vi.1986, Holzenthal, Heyn \& Armitage, 9 males (UMSP); Río Bijagual, on road to Magsasay, $10.408^{\circ} \mathrm{N}, 84.076^{\circ} \mathrm{W}$, el. $140 \mathrm{~m}, 12$. ii.1986, Holzenthal, Morse \& Fasth, 56 males (UMSP); La Selva, Agricultura Canada, 28.iv.1989, 25 males (NMNH); Limón: Río Telire and

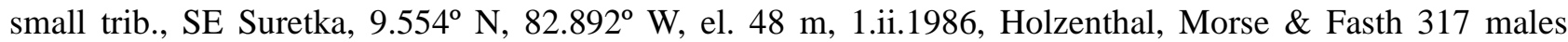
(UMSP); Río Barbilla, ca. 8 km W B-line, $10.067^{\circ} \mathrm{N}, 83.369^{\circ} \mathrm{W}$, el. $30 \mathrm{~m}$, 31.i.1986, Holzenthal, Morse \& 
Fasth, 1 male (UMSP); Río Bitey, ca. $2.5 \mathrm{~km} \mathrm{~S}$ Pandora, 9.725 $\mathrm{N}, 82.963^{\circ} \mathrm{W}$, el. $15 \mathrm{~m}$, 3.ii.1986, Holzenthal Morse \& Fasth, 5 males (UMSP); E.A.R.T.H., Río Parismina, $10.248^{\circ} \mathrm{N}, 83.570^{\circ} \mathrm{W}$, el. 5 m, 4.ii.1992, Holzenthal, Muñoz \& Kjer, 1 male (UMSP); E.A.R.T.H., forest reserve arroyo, $7.5 \mathrm{~km}$ (air) NW Pocora, $10.23^{\circ} \mathrm{N}, 83.56^{\circ} \mathrm{W}$, el. 10 m, 4-5.ii.1992, Holzenthal, Muñoz \& Kjer, 6 males (INBIO); Res. Biol. Barbilla, Río Dantas, 15 km (rd) S Pacuarito, 9.994 N, 83.443 W, el. 300 m, 27-30.i.1992, Holzenthal, Muñoz \& Kjer, 1 male (UMSP); Hacienda Tapezco, 21.iii.1999, P. Donahue et al., 1 male (NMNH); Puntarenas: Río Rincón, $6.5 \mathrm{~km}$ (air) S Rincón, 8.638 ${ }^{\circ} \mathrm{N}, 83.480^{\circ} \mathrm{W}$, el. 20 m, 7.iv.1987, Holzenthal, Hamilton \& Heyn, 1 male (UMSP); Quebrada Pita, ca. 3 km (air) W Golfito, 8.642 ${ }^{\circ}$ N, 83.193 ${ }^{\circ}$ W, el. 15 m, 15.ii.1986, Holzenthal, Morse \& Fasth, 20 males (UMSP).

\section{Ochrotrichia panamensis Flint. NEW COUNTRY RECORD}

Ochrotrichia panamensis Flint 1972:10.

The type species was described from Chiriqui, Panama.

COSTA RICA: Alajuela: Río Peje and falls, ca. $1 \mathrm{~km} \mathrm{SE} \mathrm{San} \mathrm{Vicente,} 10.277^{\circ} \mathrm{N}, 84.388^{\circ} \mathrm{W}$, el. $1450 \mathrm{~m}$, 14-15.ii.1992, Holzenthal, Muñoz \& Kjer, 6 males (UMSP); Río Toro 3.0 km (road) SW Bajos del Toro, 10.204 ${ }^{\circ} \mathrm{N}, 84.316^{\circ} \mathrm{W}$, el. $1530 \mathrm{~m}, 3-4 . i x .1990$, Holzenthal, Blahnik \& Huisman, 29 males (UMSP); same, 11.ii.1992, Holzenthal, Muñoz \& Kjer, 1 male (UMSP); Cartago: Reserva Tapantí, Río Grande de Orosi 9.686 ${ }^{\circ}$ N, 83.756 ${ }^{\circ}$ W, el. 1650 m, 18-21.iii.1987, Holzenthal, Hamilton \& Heyn, 3 males (INBIO); Reserva Tapantí, Río Dos Amigos \& falls, ca. 6 km (rd) NW tunnel, 9.704 N, 83.763º W, el. 1500 m, 4-5.viii.1990, Holzenthal, Blahnik \& Muñoz, 1 male (UMSP); Reserva Tapantí, Quebrada Segunda: @ administrtion building, $9.761^{\circ} \mathrm{N}, 83.787^{\circ} \mathrm{W}$, el. $1250 \mathrm{~m}, 9-10 . v .1990$, Holzenthal \& Blahnik, 1 male (UMSP); Puntarenas: Zona Protectora Las Tablas, Río Cotón, Sitio Cotón, 8.941 ${ }^{\circ}$ N, 82.787 W, el. 1460 m, 15.iv.1989, Holzenthal \& Blahnik, 4 males (UMSP); Río Cotón, in Las Alturas, $8.938^{\circ} \mathrm{N}, 82.826^{\circ} \mathrm{W}$, el.1360 m,16.ii1986, Holzenthal, Morse \& Fasth, 1 male (UMSP); Río Bellavista, ca. $1.5 \mathrm{~km} \mathrm{NW}$ Las Alturas, $8.951^{\circ} \mathrm{N}, 82.846^{\circ}$ W, el. 1400 m, 16-17.iii.1991, Holzenthal, Muñoz \& Huismann, 1 male (UMSP); same, 8-9.iv.1987, Holzenthal, Hamilton \& Heyn, 2 males (UMSP).

\section{Ochrotrichia quebrada Bueno-Soria \& Holzenthal}

Ochrotrichia quebrada Bueno-Soria \& Holzenthal 1998:607.

The type species was described from Guanacaste, Costa Rica.

COSTA RICA: Guanacaste: P. N. Rincón de la Vieja, Quebrada Zopilote, $10.765^{\circ} \mathrm{N}, 83.309^{\circ} \mathrm{W}$, el. 785 m, 3.iii.1986, Holzenthal \& Fasth, 1 male (UMSP).

\section{Ochrotrichia quinealensis Bueno-Soria \& Holzenthal}

Ochrotrichia quinealensis Bueno-Soria \& Holzenthal 1998:611.

The type species was described from Puntarenas, Costa Rica.

COSTA RICA: Alajuela: Reserva Forestal San Ramón, Río San Lorencito and tribs., $10.216^{\circ}$ N, $84^{\circ} .607$ W, el. 980 m, 30.iii.-1.iv.1987, Holzenthal, Hamilton \& Heyn, 2 males (UMSP); Guanacaste: Parque Nacional Guanacaste, Estación Maritza, Río Tempisquito Sur, $10.95^{\circ}$ N, $85.48^{\circ}$ W, el. 600 m, 30.viii.1990, Huisman 
\& Quesada, 4 males (UMSP); Puntarenas: Río Guineal, ca.1 Km (air) E Finca Helechales, $9.076^{\circ} \mathrm{N}, 83.092^{\circ}$ W, el. 840 m, 22.ii.1986, Holzenthal, Morse \& Fasth, 1 male (NMNH).

\section{Ochrotrichia ramona Bueno-Soria \& Holzenthal}

Ochrotrichia ramona Bueno-Soria \& Holzenthal 1998:610.

The type species was described from Alajuela, Costa Rica.

COSTA RICA: Alajuela: Reserva Forestal San Ramón, Río San Lorencito and tribs., $10.216^{\circ}$ N, 84.607 W, el. 980 m, 30.iii.-1.iv.1987, Holzenthal, Hamilton \& Heyn, 1 male (NMNH); 7 males (UMSP).

\section{Ochrotrichia silva Bueno-Soria \& Holzenthal}

Ochrotrichia silva Bueno-Soria \& Holzenthal 1998:606.

The type species was described from Alajuela, Costa Rica.

COSTA RICA: Alajuela: Reserva Forestal San Ramón, Río San Lorencito and tribs., $10.216^{\circ} \mathrm{N}, 84.607^{\circ}$ W, el. 980 m, 30.iii.-1.iv.1987, Holzenthal, Hamilton \& Heyn, 1 male (UMSP); same, 1-4.v.1990, Holzenthal \& Blahnik, 1 male (UMSP).

\section{Ochrotrichia tagala Flint. NEW COUNTRY RECORD}

Ochrotrichia tagala Flint, 1972:8.

The type species was described from Huehuetenango, Guatemala, and recorded from Nicaragua by Flint et al. (1999).

COSTA RICA: Alajuela, Río Peje and falls, ca. $1 \mathrm{~km} \mathrm{SE} \mathrm{San} \mathrm{Vicente,} 10.277^{\circ} \mathrm{N}, 84.388^{\circ} \mathrm{W}$, el. $1450 \mathrm{~m}$, 14-15.ii.1992, Holzenthal, Muñoz \& Kjer, 1 male (UMSP).

\section{Ochrotrichia tenanga (Mosely)}

Polytrichia tenanga Mosely 1937:185.

The type species was described from Chiapas, Mexico, and it has been recorded from Costa Rica, Guatemala, Honduras, Panama, Peru, and Venezuela (Flint et al. 1999).

COSTA RICA: Alajuela: Río Pizote, ca 5 km N Dos Ríos, $10.948^{\circ}$ N, 85.291º W, el. 470 m, 9.iii.1986, Holzenthal \& Fasth, 16 males (UMSP); Río Pizote, ca. 5 km (air) S Brasilia, $10.972^{\circ} \mathrm{N}, 85.345^{\circ} \mathrm{W}$, el. $390 \mathrm{~m}$, 12.iii.1986, Holzenthal \& Fast, 15 males (UMSP). Guanacaste: Río Ahogados, 10 mi.(16 km) NW Liberia, 25.vii.1965, P. J. Spangler, 1 male (NMNH); Las Cañas, 13.vii.1965, P. Spangler, 4 males (NMNH); P. N. Guanacaste, Estación Maritza, Río Tempisquito, $10.958^{\circ}$ N, 85.497 W, el. 550 m, 30-31.viii.1990, Huisman, Blahnik \& Quesada, 1 male (UMSP); Quebrada García $10.6 \mathrm{~km}$ ENE Quebrada Grande, $10,862^{\circ} \mathrm{N}, 85.428^{\circ}$ W, el. 470 m, 8.iii.1986, Holzenthal \& Fasth, 6 males (INBIO); Río Mena, $4.2 \mathrm{~km}$ Santa Cecilia, 11.059 N, 85.448 ${ }^{\circ}$ W, el. 260 m, 11.iii.1986, Holzenthal \& Fasth 5 males (UMSP); Río Tizate, 7.2 km NE, Cañas Dulces $10.773^{\circ} \mathrm{N}, 85.449^{\circ} \mathrm{W}$, el. 275 m, 28.vi.1986, Holzenthal, Heyn \& Armitage, 81 males (UMSP), 9 males 
(NMNH); Río Aguacate, $0.5 \mathrm{~km}$ E Aguacate [nr. Tronadora], 10.565 N, 84.939 W, el. $590 \mathrm{~m}$, 16.ii.1992, Holzenthal, Muñoz \& Kjer, 15 males (UMSP). Heredia: Río Bijagual, on road to Magsasay, $10.408^{\circ} \mathrm{N}$, 84.076 $\mathrm{W}$, el. $140 \mathrm{~m}, 12 . i 1.1986$, Holzenthal, Morse \& Fasth,1 male (UMSP); Estación Biológica La selva, Río Puerto Viejo, $10.440^{\circ} \mathrm{N}, 84.012^{\circ} \mathrm{W}$, el. $30 \mathrm{~m}, 19$. vi.1986, Holzenthal, Heyn \& Armitage, 1 male (INBIO); Río Sarapiquí, $7 \mathrm{~km} \mathrm{~W}$ Puerto Viejo, $10.452^{\circ} \mathrm{N}, 84.067^{\circ} \mathrm{W}$, el. $50 \mathrm{~m}$, 11.ii.1986, Morse \& Fasth, 1 male

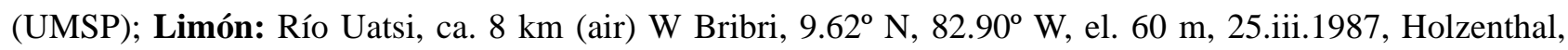
Hamilton \& Heyn, 3 males (UMSP); Río Telire and small trib., SE Suretka, 9.554 ${ }^{\circ} \mathrm{N}, 82.892^{\circ} \mathrm{W}$, el. $48 \mathrm{~m}$, 1.ii.1986, Holzenthal, Morse \& Fasth, 1 male (UMSP); Puntarenas: Río Cotón in Las Alturas, $8.938^{\circ} \mathrm{N}$, 82.826 $\mathrm{W}$, el.1360 m, 16.ii.1986, Holzenthal, Morse \& Fasth, 1 male (UMSP).

\section{Ochrotrichia vieja Bueno-Soria \& Holzenthal}

Ochrotrichia vieja Bueno-Soria \& Holzenthal 1998:608.

The type species was described from Guanacaste, Costa Rica.

COSTA RICA: Alajuela: P. N. Rincón de la Vieja, Quebrada Provisión, $10.769^{\circ}$ N, $85.281^{\circ} \mathrm{W}$, el. 810 m, 4.iii.1986, Holzethal \& Fasth, 1 male (NMNH); Puntarenas: Río Bellavista, ca. 1.5 km NW Las Alturas, 8.951 ${ }^{\circ} \mathrm{N}, 82.846^{\circ} \mathrm{W}$, el. $1400 \mathrm{~m}, 16-17$. iii.1991, Holzenthal, Muñoz \& Huisman, 1 male (UMSP); Río Cotón in Las Alturas, $8.938^{\circ} \mathrm{N}, 82.826^{\circ} \mathrm{W}, 12 . v i i i .1990$, el. $1360 \mathrm{~m}$, Holzenthal, Blahnik \& Muñoz, 1 male (UMSP).

\section{Acknowledgements}

We thank Dr. Oliver S. Flint Jr., Smithsonian Institution for his review and suggestions on the manuscript and the late Ms. Nancy Adams, Collection's Manager-Specialist, Smithsonian Institution, for her help to J. BuenoSoria during his visit to the National Museum of Natural History, Smithsonian Institution. Mr. Rafael BarbaAlvarez ably assisted in preparation of drawings and material of study. We are especially grateful to Dr. Steven C. Harris, Clarion University, for his initial work in sorting and identifying the material. Finally, we thank two anonymous reviewers for their time and effort to improve the manuscript. This material is based upon work supported by the National Science Foundation grant nos. 8512368, 8917684, 9400632, and 0117772 .

\section{Literature cited}

Botosaneanu, L. (1977) Trichoptères (imagos) de Cuba, capturés par moi-meme en 1973 (Insecta:Trichoptera). Fragmenta Entomologica, 13, 231-284.

Botosaneanu, L. (1995) Caddisflies (Trichoptera) from the Dominican Republic (West Indies), I. The Hydroptilidae. Bulletin de l' Institut Royal des Sciences Naturelles de Belgique, Entomologie, 65, 21-33.

Bueno-Soria, J. \& Holzenthal, R.W. (1998) Studies in Aquatic Insects XIV: Description of eight new species of Ochrotrichia Mosely (Trichoptera: Hydroptilidae), from Costa Rica. Proceedings of the Biological Society of Washington, 111, 604-612.

Bueno-Soria, J. \& Holzenthal, R.W. (2004) New Species of the genus Ochrotrichia Mosely (Trichoptera: Hydroptilidae) from Mexico and Panama. Transactions of the American Entomological Society, 130, 245-269.

Bueno-Soria, J. \& Santiago-Fragoso, S. (1992) Studies in aquatic insects, XI: Seven new species of the genus Ochrotrichia (Ochrotrichia) from South America (Trichoptera:Hydroptilidae). Proceedings of the Entomological Society of Washington, 94, 439-446.

Bueno-Soria, J. \& Santiago-Fragoso, S. (1997) Studies in Aquatic Insects, XII: Descriptions of nineteen new species of 
the genus Ochrotrichia Mosely (Trichoptera: Hydroptilidae) from Mexico and Central America. Proceedings of Entomological Society of Washington, 99, 359-373.

Denning, D.G. \& Blickle, R.L. (1972) A review of the genus Ochrotrichia (Trichoptera:Hydroptilidae). Annals of the Entomological Society of America, 65, 141-151.

Flint, O.S., Jr. (1964) The caddisflies (Trichoptera) of Puerto Rico. University of Puerto Rico, Agricultural Experiment Station, Technical Paper, 40, 1-80.

Flint, O.S., Jr. (1972) Studies of Neotropical Caddisflies, XIII: the Genus Ochrotrichia from Mexico and Central America (Trichoptera:Hydroptilidae). Smithsonian Contributions to Zoology 118, 1-28.

Flint, O.S., Jr. \& Bueno-Soria, J. (1999) Studies of Neotropical caddisflies LVIII: new species of the genus Ochrotrichia Mosely (Trichoptera: Hydroptilidae) from Peru. Proceedings of the Entomological Society of Washington 101, 739736.

Flint, O.S., Jr., Holzenthal, R.W. \& Harris, S.C. (1999) Catalog of the Neotropical Caddisflies (Insecta:Trichoptera). Ohio Biological Survey, Columbus, Ohio. iv+239 pp.

Mosely, M.E. (1937) Mexican Hydroptilidae (Trichoptera). The Transactions of the Royal Entomological Society of London, 86, 151-190.

Wells, A. \& Wichard, W. (1989) Caddisflies of Dominican Amber. VI. Hydroptilidae (Trichoptera). Studies on Neotropical Fauna and Environment, 24, 41-51. 